\title{
Bounds for the minimum distance function
}

\author{
Luis Núñez-Betancourt, Yuriko Pitones, and Rafael H. Villarreal
}

\begin{abstract}
Let $I$ be a homogeneous ideal in a polynomial ring $S$. In this paper, we extend the study of the asymptotic behavior of the minimum distance function $\delta_{I}$ of $I$ and give bounds for its stabilization point, $r_{I}$, when $I$ is an $F$-pure or a square-free monomial ideal. These bounds are related with the dimension and the Castelnuovo-Mumford regularity of $I$.
\end{abstract}

Keywords: Minimum distance, Castelnuovo-Mumford regularity, monomial ideal.

\section{Introduction}

In this manuscript we study the minimum distance function $\delta_{I}$ of a homogeneous ideal $I$ contained in a polynomial ring $S=\mathbb{K}\left[x_{1}, \ldots, x_{n}\right]$ over a field $\mathbb{K}$. This minimum distance function for ideals was introduced by the secondnamed and third-named authors together with Martínez-Bernal [MBPV17] to obtain an algebraic formulation of the minimum distance of projective ReedMuller-type codes over finite fields.

If $I$ is an unmixed radical graded ideal and its associate primes are generated by linear forms, then $\delta_{I}$ is non-increasing [MBPV17]. In our first result, we extend this property to any radical ideal.

Theorem A (Theorem 3.4). Suppose that $I \subseteq S$ is a radical ideal. Then, $\delta_{I}(d)$ is a non-increasing function.

Key Words: Minimum distance, Castelnuovo-Mumford regularity, monomial ideal.

2010 Mathematics Subject Classification: Primary 13D40; Secondary 13H10, 13 P25.

Received: 29.12 .2020

Accepted: 28.03.2021 
The previous result allow us to define the regularity index of $\delta_{I}, r_{I}$, as the value where $\delta_{I}$ stabilizes. If $\operatorname{dim}(S / I)=1$, previous work shows that $r_{I} \leq \operatorname{reg}(S / I)$ [GSRTR02, RMSV11], where $\operatorname{reg}(S / I)$ is the CastelnuovoMumford regularity of $S / I$. This motivated the authors to conjecture that this relation holds in greater generality.

Conjecture B ([NnBPV18]). Let $I \subseteq S$ be a radical homogeneous ideal whose associated primes are generated by linear forms. Then, $r_{I} \leq \operatorname{reg}(S / I)$.

This conjecture was previously showed for edge ideals associated to CohenMacaulay bipartite graphs [NnBPV18] and when $\operatorname{dim}(S / I)=1$ [GSRTR02, RMSV11]. However, the conjecture does not hold in general. Jaramillo and the third-named author provided an example of a monomial edge ideal $I$ such that $r_{I}>\operatorname{reg}(S / I)$ [JV21]. In this work, we find bounds for $r_{I}$ for square-free monomial ideals.

Theorem C (Theorem $4.5 \& 5.7$ ). Let $I \subseteq S$ be a square-free monomial ideal. Then, $r_{I} \leq \operatorname{dim}(S / I)$. Moreover, if $I$ is shellable or Gorenstein, then $r_{I} \leq \operatorname{reg}(S / I)$.

We also prove a similar result for ideals such that $S / I$ is a $F$-pure ring. These class of rings play an important role in the study of singularities in prime characteristic [HR76].

Theorem $\mathbf{D}$ (Theorem $5.5 \& 5.6$ ). Suppose that $\mathbb{K}$ is a field of prime characteristic. Let $I \subseteq S$ be an ideal such that $S / I$ if $F$-pure. Then, $r_{I} \leq \operatorname{dim}(S / I)$. Moreover, if $I$ is Gorenstein, then $r_{I} \leq \operatorname{reg}(S / I)$.

\section{Preliminaries}

In this section we recall some well known notion and results that are needed throughout this manuscript.

Let $S=\mathbb{K}\left[x_{1}, \ldots, x_{n}\right]=\bigoplus_{t=0}^{\infty} S_{t}$ be a polynomial ring over a field $\mathbb{K}$ with the standard grading and let $I \neq(0)$ be a homogeneous ideal of $S$. Let $d$ denote the Krull dimension of $R=S / I$.

The Hilbert function of $S / I$, denoted $H_{I}$, is given by

$$
H_{I}(t)=\operatorname{dim}_{\mathbb{K}}\left(R_{\leq t}\right)=\operatorname{dim}_{\mathbb{K}}\left(S_{\leq t} / I_{\leq t}\right)=\operatorname{dim}_{\mathbb{K}}\left(S_{\leq t}\right)-\operatorname{dim}_{\mathbb{K}}\left(I_{\leq t}\right),
$$

where $I_{\leq t}=I \cap S_{\leq t}$. By a classical theorem of Hilbert there is a unique polynomial $h_{I}(t) \in \overline{\mathbb{Q}}[t]$ of degree $d$ such that $H_{I}(t)=h_{I}(t)$ for $t \gg 0$. 
The Hilbert-Samuel multiplicity or degree of $R$, denoted e $(R)$, is the positive integer defined by $\mathrm{e}(R)=d ! \lim _{t \rightarrow \infty} H_{I}(t) / t^{d}$.

Given an integer $t \geq 1$, let $\mathcal{F}_{t}$ be the set of all zero-divisors of $S / I$ not in $I$ of degree $t \geq 1$. That is

$$
\mathcal{F}_{t}=\left\{f \in S_{t} \mid f \notin I,(I: f) \neq I\right\} .
$$

We note that $(I: f) \neq I$ is equivalent to $f \in \mathfrak{p}$ for some $\mathfrak{p} \in \operatorname{Ass}_{S}(S / I)$, $A s s_{S}(S / I)$ is the set of associated primes of $S / I$.

Definition 2.1. The minimum distance function of $I$ is the function $\delta_{I}: \mathbb{N}_{+} \rightarrow$ $\mathbb{Z}$ given by

$$
\delta_{I}(t)= \begin{cases}\mathrm{e}(S / I)-\max \left\{\mathrm{e}(S /(I, f)) \mid f \in \mathcal{F}_{t}\right\} & \text { if } \mathcal{F}_{t} \neq \emptyset \\ \mathrm{e}(S / I) & \text { if } \mathcal{F}_{t}=\emptyset\end{cases}
$$

Definition 2.2. Let $I \subseteq S$ be a graded ideal and let $\mathbb{F}_{\star}$ be the minimal graded free resolution of $S / I$ as an $S$-module:

$$
\mathbb{F}_{\star}: \quad 0 \rightarrow \bigoplus_{j} S(-j)^{\beta_{g j}} \rightarrow \cdots \rightarrow \bigoplus_{j} S(-j)^{\beta_{1 j}} \rightarrow S \rightarrow S / I \rightarrow 0 .
$$

The Castelnuovo-Mumford regularity of $S / I$, regularity of $S / I$ for short, is defined as

$$
\operatorname{reg}(S / I)=\max \left\{j-i \mid \beta_{i j} \neq 0\right\} .
$$

The following result shows the asymptotic behavior of $\delta_{I}$ for a particular case of graded ideals.

An ideal $I \subseteq S$ is called unmixed if all its associated primes have the same height, in other case $I$ is mixed.

Theorem 2.3 ([MBPV17, Theorem 3.8]). Let $I \subseteq S$ be an unmixed radical homogeneous ideal. If all the associated primes of I are generated by linear forms, then there is an integer $r_{0} \geq 1$ such that

$$
\delta_{I}(1)>\delta_{I}(2)>\cdots>\delta_{I}\left(r_{0}\right)=\delta_{I}(d)=1 \text { for } d \geq r_{0} .
$$

The integer $r_{0}$ where the stabilization occurs is called the regularity index of $\delta_{I}$ and is denoted by $r_{I}$. In Section 3, we show that one can define this index for any radical ideal. 
Local cohomology Let $R$ be a commutative Noetherian ring with identity and let $I$ be a homogeneous ideal generated by the forms $f_{1}, \ldots, f_{\ell} \in R$. Consider the Čech complex, $\check{\mathrm{C}}^{\star}(\bar{f} ; R)$ :

$$
0 \rightarrow R \rightarrow \bigoplus_{i} R_{f_{i}} \rightarrow \bigoplus_{i, j} R_{f_{i} f_{j}} \rightarrow \cdots \rightarrow R_{f_{1}, \ldots, f_{\ell}} \rightarrow 0
$$

where $\check{\mathrm{C}}^{i}(\bar{f} ; R)=\bigoplus_{1 \leq j_{1} \leq \ldots \leq j_{i} \leq \ell} R_{f_{j_{1}}, \ldots, f_{j_{i}}}$ and the homomorphism in every summand is a localization map with appropriate sign.

Definition 2.4. Let $M$ be a graded $R$-modue. The $i$-th local cohomology of $M$ with support in $I$ is defined as

$$
H_{I}^{i}(M)=H^{i}\left(\check{\mathrm{C}}^{\star}(\bar{f} ; R) \otimes_{R} M\right) .
$$

Remark 2.5. Since $M$ is a graded $R$-module and $I$ is homogeneous the local cohomology module $H_{I}^{i}(M)$ is graded.

Remark 2.6. If $\phi: M \rightarrow N$ is a homogeneous $R$-module homomorphism of degree $t$, then the induced $R$-module map $H_{I}^{i}(M) \rightarrow H_{I}^{i}(N)$ is homogeneous of degree $t$.

Theorem 2.7 (Grothendieck's Vanishing Theorem). Let $M$ be an $R$-module of dimension $d$. Then, $H_{I}^{i}(M)=0$ for all $i>d$.

Theorem 2.8 (Grothendieck's Non-Vanishing Theorem). Let $M$ be a finitely generated $R$-module of dimension $d$. Then, $H_{I}^{d}(M) \neq 0$.

Definition 2.9. Let $M$ be an $R$-module with dimension $d$. The $a_{i}$-invariants, $a_{i}(M)$, for $i=0, \ldots, d$ are defined as follows. If $H_{\mathfrak{m}}^{i}(M) \neq 0$,

$$
a_{i}(M)=\max \left\{\alpha \mid H_{\mathfrak{m}}^{i}(M)_{\alpha} \neq 0\right\}
$$

for $0 \leq i \leq d$, where $H_{\mathfrak{m}}^{i}(M)$ denotes the local cohomology module with support in the maximal ideal $\mathfrak{m}$. If $H_{\mathfrak{m}}^{i}(M)=0$, we set $a_{i}(M)=-\infty$.

If $d=\operatorname{dim}(M)$, then, $a_{d}(M)$, is often just called the $a$-invariant of $M$.

The $a$-invariant, is a classical invariant [GW78], and is closely related to the Castelnuovo-Mumford regularity.

Definition 2.10. Let $R$ be a positively graded ring and let $M$ be a finitely generated $R$-module. The Castelnuovo-Mumford regularity of $M, \operatorname{reg}(M)$, is defined as

$$
\operatorname{reg}(M)=\max \left\{a_{i}(M)+i \mid 1 \leq i \leq d\right\}
$$


Remark 2.11. If $M$ is a standard graded module of dimension d, then the ainvariant is related to the Castelnuovo-Mumford regularity, via the inequeality $a(M)+d \leq \operatorname{reg}(M)$ which is equality in the case Cohen-Macaulay.

Definition 2.12. Suppose that $R$ has prime characteristic $p$. The Frobenius map $F: R \rightarrow R$ is defined by $r \mapsto r^{p}$.

Remark 2.13. If $R$ is reduced, $R^{1 / p^{e}}$ the ring of the $p^{e}$-th roots of $R$ is well defined, and $R \subseteq R^{1 / p^{e}}$.

\section{Asymptotic behavior of the minimum distance func- tion}

In this section we prove that the minimum distance function $\delta_{I}$ is non-increasing. Then, the notion of regularity index of $\delta_{I}$ is well defined. We also find what is the stable value of the minimum distance function. We start this section establishing notation.

Notation 3.1. Given an ideal $I \subseteq S$, we set

$$
\begin{aligned}
& \mathcal{A}(I)=\left\{\mathfrak{p} \in \operatorname{Ass}_{S}(S / I) \mid \operatorname{dim}(S / I)=\operatorname{dim}(S / \mathfrak{p})\right\} ; \\
& \mathcal{V}(I)=\{\mathfrak{p} \in \operatorname{Spec}(S) \mid I \subseteq \mathfrak{p}\} \\
& \mathcal{D}(I)=\operatorname{Spec}(S) \backslash \mathcal{V}(I) .
\end{aligned}
$$

Remark 3.2. For an ideal $I \subseteq S$, we have

$$
\mathrm{e}(S / I)=\sum_{\mathfrak{p} \in \mathcal{A}(I)} \lambda_{S_{\mathfrak{p}}}\left(S_{\mathfrak{p}} / I S_{\mathfrak{p}}\right) \mathrm{e}(S / \mathfrak{p}),
$$

where $\lambda_{S_{\mathfrak{p}}}\left(S_{\mathfrak{p}} / I S_{\mathfrak{p}}\right)$ denotes the length of $S_{\mathfrak{p}} / I S_{\mathfrak{p}}$ as $S_{\mathfrak{p}}$-module, by the additivity formula (see for instance [HS06, Theorem 11.2.4]). In particular, if I is radical, then

$$
\mathrm{e}(S / I)=\sum_{\mathfrak{p} \in \mathcal{A}(I)} \mathrm{e}(S / \mathfrak{p})
$$

Lemma 3.3. Suppose that $I$ is a radical ideal. Let $f \in \mathcal{F}_{t}$ such that $\operatorname{dim}(S /(I, f))$ $=\operatorname{dim}(S / I)$. Then, $\mathcal{A}((I, f))=\mathcal{A}(I) \cap \mathcal{V}(f)$. Furthermore,

$$
\mathrm{e}(S /(I, f))=\sum_{\mathfrak{p} \in \mathcal{A}(I) \cap \mathcal{V}(f)} \mathrm{e}(S / \mathfrak{p}) .
$$

Proof. Let $J=(I, f)$. Let $Q$ be an associated prime of $J$. Since $I \subseteq J$, there exists an associated prime $\mathfrak{p}$ of $I$ such that $\mathfrak{p} \subseteq Q$. If $\operatorname{dim}(S / Q)=\operatorname{dim}(S / J)=$ $\operatorname{dim}(S / I)$, then $\mathfrak{p}=Q$. Thus, $\mathcal{A}(J) \subseteq \mathcal{A}(I) \cap \mathcal{V}(f)$. 
Let $Q \in \mathcal{A}(I) \cap \mathcal{V}(f)$. Then, $J \subseteq Q$ and $\operatorname{dim}(S / Q)=\operatorname{dim}(S / J)$. Then, $Q$ is a minimal prime of $S / J$. Thus, $Q \in \operatorname{Ass}_{S}(S / J)$, and so, $Q \in \mathcal{A}(J)$.

We note that $J$ is not necessarily radical. However, $J S_{\mathfrak{p}}=I S_{\mathfrak{p}}$ for every $\mathfrak{p} \in \mathcal{A}(I) \cap \mathcal{V}(f)$. Thus, $\lambda_{S_{\mathfrak{p}}}\left(S_{\mathfrak{p}} / I S_{\mathfrak{p}}\right)=1$ for every $\mathfrak{p} \in \mathcal{A}(I) \cap \mathcal{V}(f)$. Then,

$$
\mathrm{e}(S /(I, f))=\sum_{\mathfrak{p} \in \mathcal{A}(I) \cap \mathcal{V}(f)} \mathrm{e}(S / \mathfrak{p})
$$

by the additivity formula.

We now show that the minimum distance function is non-increasing.

Theorem 3.4. Suppose that $I$ is a radical ideal. Then, $\delta_{I}(d)$ is a nonincreasing function.

Proof. If $\mathcal{F}_{t}=\emptyset$ for every $t \geq 1$, then $\delta_{I}(t)=\mathrm{e}(S / I)$, which is the maximum value. We note that this case is equivalent to $I$ being a prime ideal.

We now assume that $\mathcal{F}_{t} \neq \emptyset$ for some $t \in \mathbb{N}$. We note that in this case $\operatorname{dim}(S / I) \neq 0$, otherwise, $I=\mathfrak{m}$ and so $\mathcal{F}_{t}=\emptyset$. Let $f \in \mathcal{F}_{t}$ such that $\delta_{I}(t)=\mathrm{e}(S / I)-\mathrm{e}(S /(I, f))$. Then,

$$
\begin{aligned}
\delta_{I}(t) & =\mathrm{e}(S / I)-\mathrm{e}(S /(I, f)) \\
& =\sum_{\mathfrak{p} \in \mathcal{A}(I)} \mathrm{e}(S / \mathfrak{p})-\sum_{\mathfrak{p} \in \mathcal{A}((I, f))} \mathrm{e}(S / \mathfrak{p}) \\
& =\sum_{\mathfrak{p} \in \mathcal{A}(I)} \mathrm{e}(S / \mathfrak{p})-\sum_{\mathfrak{p} \in \mathcal{A}(I) \cap \mathcal{V}(f)} \mathrm{e}(S / \mathfrak{p}) \\
& =\sum_{\mathfrak{p} \in \mathcal{A}(I) \cap \mathcal{D}(f)} \mathrm{e}(S / \mathfrak{p}) .
\end{aligned}
$$

Since $I$ is radical and $\operatorname{dim}(S / I)>0$, we have that $\mathfrak{m}$ is not an associated prime. Then, $\mathfrak{m} f \nsubseteq I$ because $f \notin I$. We conclude that there exists $i=1, \ldots, n$ such that $x_{i} f \notin I$. In particular, $x_{i} f \in \mathcal{F}_{t+1}$ and $\mathcal{F}_{t+1} \neq \emptyset$. Then,

$$
\begin{aligned}
\delta_{I}(t+1) & \leq \sum_{\mathfrak{p} \in \mathcal{A}(I) \cap \mathcal{D}\left(x_{i} f\right)} \mathrm{e}(S / \mathfrak{p}) \\
& =\sum_{\mathfrak{p} \in \mathcal{A}(I) \cap \mathcal{D}\left(x_{i}\right) \cap \mathcal{D}(f)} \mathrm{e}(S / \mathfrak{p}) \\
& \leq \sum_{\mathfrak{p} \in \mathcal{A}(I) \cap \mathcal{D}(f)} \mathrm{e}(S / \mathfrak{p})=\delta_{I}(t) .
\end{aligned}
$$


Thanks to the previous theorem we have that the minimum distance function eventually stabilizes, and it has a regularity index.

Definition 3.5. Suppose that $I$ is a radical ideal. The regularity index of $\delta_{I}$, denoted by $r_{I}$, is defined by

$$
r_{I}=\min \left\{s \in \mathbb{N} \mid \delta_{I}(s)=\lim _{t \rightarrow \infty} \delta_{I}(t)\right\}
$$

Proposition 3.6. Suppose that $I$ is a radical ideal. Then,

$$
\delta_{I}(t)=\min \left\{\mathrm{e}(S / \mathfrak{p}) \mid \mathfrak{p} \in \operatorname{Ass}_{S}(S / I)\right\}
$$

for $t \gg 0$ if $I$ is unmixed and $\delta_{I}(t)=0$ for $t \gg 0$ otherwise.

Proof. We first assume that $I$ is mixed. Let $J_{1}$ be the intersection of the minimal primes of $I$ of $\operatorname{dimension} \operatorname{dim}(S / I)$ and let $J_{2}$ be the intersection of the minimal primes of $I$ of dimension smaller than $\operatorname{dim}(S / I)$. Let $f \in J_{1} \backslash I$. Let $\alpha=\operatorname{deg}(f)$. In particular, $f \in \mathcal{F}_{\alpha}$. We note that $\operatorname{dim}(S / I)=\operatorname{dim}(S /(I, f))$ and $\mathcal{A}(I)=\mathcal{A}(I, f)$, and so, $\mathrm{e}(S /(I, f))=\mathrm{e}(S / I)$. We conclude that $\delta_{I}(t)=0$. Since $\delta_{I}$ is nondecresing by Theorem 3.4, we obtain that $\delta_{I}(t)=0$ for $t \geq \alpha$.

We now assume that $I$ is unmixed. Then, $\mathcal{A}(I)=\operatorname{Ass}_{S}(S / I)$. If $I$ is a prime ideal, then $\delta_{I}(t)=\mathrm{e}(S / I)$ for every $t \in \mathbb{N}$, and our claim follows. We assume that $\operatorname{dim}(S / I)>0$ and that $I$ is not a prime ideal. For every $f \in \mathcal{F}_{t}$, there exists a prime ideal $\mathfrak{p}$ such that $f \notin \mathfrak{p}$. Then,

$$
\mathrm{e}(S / I)-\mathrm{e}(S /(I, f)) \geq \mathrm{e}(S / \mathfrak{p}) \geq \min \left\{\mathrm{e}(S / \mathfrak{p}) \mid \mathfrak{p} \in \operatorname{Ass}_{S}(S / I)\right\}
$$

We conclude that $\delta_{I}(t) \geq \min \left\{\mathrm{e}(S / \mathfrak{p}) \mid \mathfrak{p} \in \operatorname{Ass}_{S}(S / I)\right\}$. Let $\mathfrak{p}_{1}, \ldots, \mathfrak{p}_{\ell}$ denote the associated primes of $I$ in an order such that $e\left(S / \mathfrak{p}_{i}\right) \leq \mathrm{e}\left(S / \mathfrak{p}_{j}\right)$ for $i \leq j$. Let $f \in \mathfrak{p}_{2} \cap \ldots \cap \mathfrak{p}_{\ell} \backslash I$. Let $\alpha=\operatorname{deg}(f)$. We have that $f \in \mathcal{F}_{\alpha}$. Then, $\delta_{I}(\alpha) \leq \mathrm{e}(S / I)-\mathrm{e}(S /(I, f))=\mathrm{e}\left(S / \mathfrak{p}_{1}\right)$. Since $\delta$ is nondecresing by Theorem 3.4 , we obtain that $\delta_{I}(t) \leq \mathrm{e}\left(S / \mathfrak{p}_{1}\right)$ for $t \geq \alpha$. We conclude that $\delta_{I}(t)=\mathrm{e}\left(S / \mathfrak{p}_{1}\right)$ for $t \geq \alpha$.

Proposition 3.7. Let $I$ be a mixed radical ideal. Let $J_{1}$ be the intersection of the minimal primes of $I$ of dimension $\operatorname{dim}(S / I)$ and let $J_{2}$ be the intersection of the minimal primes of $I$ of dimension smaller than $\operatorname{dim}(S / I)$. Then, $r_{I}=$ $\min \left\{t \mid\left[J_{1} / I\right]_{t} \neq 0\right\}$.

Proof. As in the proof of Proposition 3.6, we have that $\delta_{I}(t)=0$ for $t \geq$ $\min \left\{t \mid\left[J_{1} / I\right]_{t} \neq 0\right\}$. We conclude that $r_{I} \leq \min \left\{t \mid\left[J_{1} / I\right]_{t} \neq 0\right\}$.

Let $f \in\left(\bigcup_{\mathfrak{p} \in \operatorname{Ass}_{S}(S / I)} \mathfrak{p}\right) \backslash I$ of degree strictly less than $\min \left\{t \mid\left[J_{1} / I\right]_{t} \neq\right.$ $0\}$. Then, $f \notin J_{1}$, and so, there exists a prime ideal $\mathfrak{p}$ such that $f \notin \mathfrak{p}$ and 
$\operatorname{dim}(S / \mathfrak{p})=\operatorname{dim}(S / I)$. Then, $e(S / I)-\mathrm{e}(S /(I, f)) \geq \mathrm{e}(S / \mathfrak{p})$. We conclude that $\delta_{I}(t)>0$. Then, $r_{I} \geq \min \left\{t \mid\left[J_{1} / I\right]_{t} \neq 0\right\}$.

Proposition 3.8. Suppose that $I \subset S$ is an unmixed radical ideal with associated primes $\mathfrak{p}_{1}, \ldots, \mathfrak{p}_{r}$ and $\mathfrak{q}_{1}, \ldots, \mathfrak{q}_{s}$ such that $\mathrm{e}\left(S / \mathfrak{p}_{i}\right)=\min \{\mathrm{e}(S / Q) \mid Q \in$ $\left.\operatorname{Ass}_{S}(S / I)\right\}$. And let $J_{i}=\left(\bigcap_{j \neq i} \mathfrak{p}_{j}\right) \cap\left(\bigcap_{j=1}^{s} \mathfrak{q}_{j}\right)$. Then, $r_{I}=\min \{t \mid \exists i$ such that $\left.\left[J_{i} / I\right]_{t} \neq 0\right\}$.

Proof. Set e $=\mathrm{e}\left(S / \mathfrak{p}_{i}\right)$. We have that $\delta_{I}(t)=e$, for $t \geq \min \{t \mid \exists i$ such that $\left.\left[J_{i} / I\right]_{t} \neq 0\right\}$, as in the proof of Proposition 3.6. We conclude that $r_{I} \leq$ $\min \left\{t \mid \exists i\right.$ such that $\left.\left[J_{i} / I\right]_{t} \neq 0\right\}$.

Let $f \in\left(\bigcup_{\mathfrak{p} \in \operatorname{Ass}_{S}(S / I)} \mathfrak{p}\right) \backslash I$ of degree strictly less than $\min \{t \mid \exists i$ such that $\left.\left[J_{i} / I\right]_{t} \neq 0\right\}$. Then, $f \notin J_{i}$ for every $i$, and so, either $f \notin \mathfrak{q}_{j}$ or $f$ does not belong to two different primes $\mathfrak{p}_{i}$ and $\mathfrak{p}_{j}$. In both cases, $\operatorname{dim}(S / I)=$ $\operatorname{dim}(S /(I, f))$. In the first case, $\mathrm{e}(S / I)-\mathrm{e}(S /(I, f)) \geq \mathrm{e}\left(S / \mathfrak{q}_{j}\right)>e$. In the second case, e $(S / I)-\mathrm{e}(S /(I, f)) \geq 2 \mathrm{e}>\mathrm{e}$. We conclude that $\delta_{I}(t)>\mathrm{e}$. Then, $r_{I} \geq \min \left\{t \mid \exists i\right.$ such that $\left.\left[J_{i} / I\right]_{t} \neq 0\right\}$.

\section{Stanley-Reisner ideals associated to a shellable simpli- cial complex}

In this section we use the shellability condition to relate the regularity index of a Stanley-Reisner ideal of a shellable simplicial complex, $I_{\Delta}$, with the Castelnuovo-Mumford regularity.

Definition 4.1. A simplicial complex on a vertex set $X=\left\{x_{1}, x_{2}, \ldots, x_{n}\right\}$ is a collection of subsets of $X$, called faces, satisfying that $\left\{x_{i}\right\} \in \Delta$ for every $i \in[n]$ and, if $\sigma \in \Delta$ and $\theta \subseteq \sigma$ then $\theta \in \Delta$. A face of $\Delta$ not properly contained in another face of $\Delta$ is called a facet.

A face $\sigma \in \Delta$ of cardinality $|\sigma|=i+1$ has dimension $i$ and is called an $i$-face of $\Delta$. The dimension of $\Delta$ is $\operatorname{dim} \Delta=\max \{\operatorname{dim} \sigma \mid \sigma \in \Delta\}$, or if $\Delta=\{\}$ is the void complex, which has no faces. We say that $\Delta$ is pure if all its facets have the same dimension.

Let $\Delta$ be a simplicial complex of dimension $d$ with the vertex set $[n]=$ $\{1,2, \ldots, n\}$, and let $\mathbb{K}$ be a field. The square-free monomial ideal $I_{\Delta}$ in the polynomial ring $S=\mathbb{K}\left[x_{1}, \ldots, x_{n}\right]$ is generated by the monomials $x^{\sigma}=\prod_{i \in \sigma} x_{i}$ which $\sigma$ is a non-face in $\Delta$. 
The simplicial complex $\Delta$ is said Cohen-Macaulay when the quotient ring $\mathbb{K}[\Delta]=S / I_{\Delta}$, called Stanley-Reisner ring of $\Delta$, is Cohen-Macaulay.

Definition 4.2. A pure simplicial complex $\Delta$ of dimension $d$ is shellable if the facets of $\Delta$ can be order $\sigma_{1}, \ldots, \sigma_{s}$ such that

$$
\bar{\sigma}_{i} \bigcap\left(\bigcup_{j=1}^{i-1} \bar{\sigma}_{j}\right)
$$

is pure of dimension $d-1$ for all $i \geq 2$. Here $\bar{\sigma}_{i}=\left\{\sigma \in \Delta \mid \sigma \subseteq \sigma_{i}\right\}$. If $\Delta$ is pure shellable, $\sigma_{1}, \ldots, \sigma_{s}$ is called a shelling.

Theorem 4.3 ([Vil15, Theorem 6.3.23]). Let $\Delta$ be a simplicial complex. If $\Delta$ is pure shellable, then $\Delta$ is Cohen-Macaulay over any field $\mathbb{K}$.

Remark 4.4. Let $\Delta$ be a simplicial complex. Suppose that $\Delta$ is pure shellable with ordered facets $\sigma_{1}, \ldots, \sigma_{s}$. We have that $\sigma_{1}, \ldots, \sigma_{i}$ is a shelling for the simplicial complex associated to $\sigma_{1} \cup \ldots \cup \sigma_{i}$. Let $P_{\sigma_{j}}=\left(x_{t} \mid t \notin \sigma_{j}\right)$. Then,

$$
S / P_{\sigma_{1}} \cap \ldots \cap P_{\sigma_{i}}
$$

is Cohen.Macaulay for every $i=1, \ldots s$ by Theorem 4.3.

We are now able to show one of our main results.

Theorem 4.5. Let $I=I_{\Delta}$ be the Stanley-Reisner ideal of a shellable simplicial complex, with $\operatorname{dim}\left(S / I_{\Delta}\right)=d$. Then $r_{I} \leq \operatorname{reg}(S / I)$.

Proof. Let $d=\operatorname{dim}\left(S / I_{\Delta}\right)$. Since $\Delta$ is shellable, $S / I_{\Delta}$ is a Cohen-Macaulay ring by Theorem 4.3. Let $\mathfrak{p}_{1}, \ldots, \mathfrak{p}_{\ell}$ denote the associate primes of $I$. For $1 \leq i \leq \ell$, we set $R_{i}=S / \mathfrak{p}_{1} \cap \mathfrak{p}_{2} \cap \cdots \cap \mathfrak{p}_{i}$ and $J_{i}=\mathfrak{p}_{1} \cap \cdots \cap \mathfrak{p}_{i}$. We have that $R_{i}$ is Cohen-Macaulay of dimension $d$, because $J_{i}$ is a shelling of $I_{\Delta}$.

We have the following short exact sequence;

$$
0 \longrightarrow J_{i-1} / J_{i} \longrightarrow R_{i} \longrightarrow R_{i-1} \longrightarrow 0
$$

for $2 \leq i \leq \ell$.

We note that $\operatorname{dim}\left(R_{i-1}\right)=d$, and so, $H_{\mathfrak{m}}^{j}\left(R_{i-1}\right)=0$ for all $j>d$. Then, the short exact sequence induces a long exact sequence as follows:

$$
\begin{aligned}
0 & \rightarrow H_{\mathfrak{m}}^{0}\left(J_{i-1} / J_{i}\right) \rightarrow H_{\mathfrak{m}}^{0}\left(R_{i}\right) \rightarrow H_{\mathfrak{m}}^{0}\left(R_{i-1}\right) \rightarrow \cdots \\
& \rightarrow H_{\mathfrak{m}}^{d}\left(J_{i-1} / J_{i}\right) \rightarrow H_{\mathfrak{m}}^{d}\left(R_{i}\right) \rightarrow H_{\mathfrak{m}}^{d}\left(R_{i-1}\right) \rightarrow 0 .
\end{aligned}
$$


Since $R_{i-1}$ and $R_{i}$ are Cohen-Macaulay rings, we have that a short exact sequence reduces to

$$
0 \rightarrow H_{\mathfrak{m}}^{d}\left(J_{i-1} / J_{i}\right) \rightarrow H_{\mathfrak{m}}^{d}\left(R_{i}\right) \rightarrow H_{\mathfrak{m}}^{d}\left(R_{i-1}\right) \rightarrow 0,
$$

because all the other local cohomology modules vanish. Then, $J_{i-1} / J_{i}$ is also Cohen-Macaulay of dimension $d$. We have that $a_{d}\left(J_{i-1} / J_{i}\right) \leq a_{d}\left(R_{i}\right)$, and

$$
\operatorname{reg}\left(J_{i-1} / J_{i}\right)=a_{d}\left(J_{i-1} / J_{i}\right)+d \leq a_{d}\left(R_{i}\right)+d=\operatorname{reg}\left(R_{i}\right) .
$$

for $2 \leq i \leq \ell$. By Proposition 3.8,

$$
r_{I} \leq \min \left\{t \mid\left[J_{\ell-1} / J_{\ell}\right]_{t} \neq 0\right\} \leq \operatorname{reg}\left(J_{\ell-1} / J_{\ell}\right) .
$$

Then, $r_{I} \leq \operatorname{reg}\left(R_{\ell}\right)=\operatorname{reg}\left(S / I_{\Delta}\right)$.

\section{Results related to $F$-purity}

Definition 5.1. Let $R$ be a Noetherian ring of prime characteristic $p$, and $F: R \rightarrow R$ be the Frobenius map. We say that $R$ is $F$-pure if for every $R$-module, $M$, we have that

$$
M \otimes_{R} R \stackrel{1_{M} \otimes_{R} F}{>} M \otimes_{R} R
$$

is injective. We say that $R$ is $F$-finite if $R$ is finitely generated as $R^{p}$-module.

Definition 5.2. Suppose that $\mathbb{K}$ has prime characteristic, $\mathbb{K}$ is $F$-finite, and that $I$ is a radical ideal. Then, we set

- $F_{*}^{e} S / I:=\left\{F_{*}^{e} f \mid f \in S / I\right\} \cong S / I$ as a Abelian groups, but the action of $S / I$ on $F_{*}^{e} S / I$ : is given by $r F_{*}^{e} f=F_{*}^{e} f^{p^{e}} f$.

- $\mathfrak{m}_{e}=\left\{f \in S / I \mid \phi\left(F_{*}^{e} f\right) \in \mathfrak{m} \forall \phi: F_{*}^{e} S / I \rightarrow S / I\right\}[$ AE05].

- $b_{e}=\max \left\{t \mid \mathfrak{m}^{t} \nsubseteq \mathfrak{m}_{e}\right\}$.

- $\operatorname{fpt}(S / I)=\lim _{e \rightarrow \infty} \frac{b_{e}}{p^{e}}[\mathrm{TW} 04]$.

Theorem 5.3 ([DSNnB18, Theorem B]). Suppose that $\mathbb{K}$ has prime characteristic. If $S / I$ is a $F$-pure ring, then $a_{i}(S / I) \leq-\operatorname{fpt}(S / I)$. Furthermore, if $S / I$ is a Gorenstein ring, then $\operatorname{reg}(S / I)=\operatorname{dim}(S / I)-\operatorname{fpt}(S / I)$.

Remark 5.4. Suppose that $\mathbb{K}$ has prime characteristic, $\mathbb{K}$ is F-finite, and that $S / I$ is a F-pure ring. Let $\mathfrak{p}_{1}, \ldots, \mathfrak{p}_{\ell}$ be the minimal primes of $I$, and $J_{i}=\bigcap_{i \neq j} \mathfrak{p}_{j}$. Then, $S / J_{i}$ is F-pure [Sch10, Corollary 4.8]. Furthermore, $\operatorname{fpt}(S / I) \leq \operatorname{fpt}\left(S / J_{i}\right)\left[D S N n B 18\right.$, Theorem 4.7], because $J_{i} \cdot S / I$ is a compatible ideal for $S / I$. 
Theorem 5.5. Suppose that $\mathbb{K}$ has prime characteristic. If $S / I$ is a F-pure ring, then $r_{I} \leq \operatorname{dim}(S / I)$.

Proof. Let $\mathfrak{p}_{1}, \ldots, \mathfrak{p}_{\ell}$ be the minimal primes of $I$. For $i=1, \ldots, \ell$, we set $J_{i}=\bigcap_{i \neq j} \mathfrak{p}_{j}$. We have a short exact sequence

$$
0 \rightarrow J_{i} / I \rightarrow S / I \rightarrow S / J_{i} \rightarrow 0 .
$$

This induces a long exact sequence

$$
0 \rightarrow H_{\mathfrak{m}}^{0}\left(J_{i} / I\right) \rightarrow H_{\mathfrak{m}}^{0}(S / I) \rightarrow H_{\mathfrak{m}}^{0}\left(S / J_{i}\right) \rightarrow H_{\mathfrak{m}}^{1}\left(J_{i} / I\right) \rightarrow \ldots
$$

Since both $S / J_{i}$ and $S / I$ are $F$-pure, we have that $a_{j}\left(S / J_{i}\right) \leq 0$ and $a_{j}(S / I) \leq$ 0 for every $j$. Then, $a_{j}\left(J_{i} / I\right) \leq 0$ for every $j$ [HR76, Proposition 2.4]. Then,

$$
\begin{aligned}
\min \left\{t \mid\left[J_{i} / I\right]_{t} \neq 0\right\} & \leq \max \left\{\ell \mid \beta_{0, \ell}\left(J_{i} / I\right) \neq 0\right\} \\
& \leq \operatorname{reg}\left(J_{i} / I\right) \\
& =\max \left\{a_{j}\left(J_{i} / I\right)+j\right\} \\
& \leq \operatorname{dim}(S / I) .
\end{aligned}
$$

Theorem 5.6. Suppose that $\mathbb{K}$ has prime characteristic. If $S / I$ is a F-pure ring and Gorenstein, then $r_{I} \leq \operatorname{reg}(S / I)$.

Proof. We first assume that $\mathbb{K}$ is $F$-finite. Let $\mathfrak{p}_{1}, \ldots, \mathfrak{p}_{\ell}$ be the minimal primes of $I$. For $i=1, \ldots, \ell$, we set $J_{i}=\bigcap_{i \neq j} \mathfrak{p}_{j}$. We have a short exact sequence

$$
0 \rightarrow J_{i} / I \rightarrow S / I \rightarrow S / J_{i} \rightarrow 0 \text {. }
$$

This induces a long exact sequence

$$
0 \rightarrow H_{\mathfrak{m}}^{0}\left(J_{i} / I\right) \rightarrow H_{\mathfrak{m}}^{0}(S / I) \rightarrow H_{\mathfrak{m}}^{0}\left(S / J_{i}\right) \rightarrow H_{\mathfrak{m}}^{1}\left(J_{i} / I\right) \rightarrow \ldots
$$

Since both $S / J_{i}$ and $S / I$ are $F$-pure, we have that $a_{j}\left(S / J_{i}\right) \leq-\operatorname{fpt}\left(S / J_{i}\right)$ and $a_{j}(S / I) \leq-\operatorname{fpt}(S / I)$ for every $j$. Then,

$$
a_{j}\left(J_{i} / I\right) \leq \max \left\{-\operatorname{fpt}\left(S / J_{i}\right),-\operatorname{fpt}(S / I)\right\} \leq-\operatorname{fpt}(S / I)
$$

for every $j$ by Theorem 5.3. Then,

$$
\begin{aligned}
\min \left\{t \mid\left[J_{i} / I\right]_{t} \neq 0\right\} & \leq \max \left\{\ell \mid \beta_{0, \ell}\left(J_{i} / I\right) \neq 0\right\} \\
& \leq \operatorname{reg}\left(J_{i} / I\right) \\
& =\max \left\{a_{j}\left(J_{i} / I\right)+j\right\} \\
& =\max \{-\operatorname{fpt}(S / I)+j\} \\
& \leq \operatorname{dim}(S / I)-\operatorname{fpt}(S / I) \\
& =\operatorname{reg}(S / I) \text { by Theorem } 5.3 .
\end{aligned}
$$


The result for non $F$-finite fields follows from taking the product $\otimes_{\mathbb{K}} \overline{\mathbb{K}}$, because the numerical invariants do not change after field extensions. In addition, $F$ purity is stable for field extensions.

Theorem 5.7. Let $\mathbb{K}$ be any field and $I$ is a square-free monomial ideal. Then, $r_{I} \leq \operatorname{dim}(S / I)$. If $S / I$ is a Gorenstein ring, then $r_{I} \leq \operatorname{reg}(S / I)$.

Proof. If $\mathbb{K}$ has prime characteristic, the result follows from Theorems 5.5 and 5.6.

We now assume that $\mathbb{K}$ has characteristic zero. Since field extensions do not affect whether a ring is Gorenstein and their dimension, without loss of generality we can assume that $\mathbb{K}=\mathbb{Q}$. Let $A=\mathbb{Z}\left[x_{1}, \ldots, x_{n}\right]$ and $I_{A}$ the monomial ideal generated by the monomials in $I$. We have that $r_{I}=$ $r_{I_{A} \otimes_{\mathbb{Z}} \mathbb{F}_{p}}$ by Propositions 3.7 and 3.8, since $\operatorname{dim}(S / I)=\operatorname{dim}\left(A \otimes_{\mathbb{Z}} \mathbb{Q} / I_{A} \otimes_{\mathbb{Z}} \mathbb{Q}\right)=$ $\operatorname{dim}\left(\mathbb{F}_{p}\left[x_{1}, \ldots, x_{n}\right] / I_{A} \otimes_{\mathbb{Z}} \mathbb{F}_{p}\right)$. Then,

$$
r_{I}=r_{I_{A} \otimes_{\mathbb{Z}} \mathbb{F}_{p}} \leq \operatorname{dim}\left(\mathbb{F}_{p}\left[x_{1}, \ldots, x_{n}\right] / I_{A} \otimes_{\mathbb{Z}} \mathbb{F}_{p}\right)=\operatorname{reg}(S / I)
$$

by Theorem 5.6, because Stanley-Reisner rings in prime characteristic are $F$-pure.

We have that

$$
\operatorname{reg}_{S}(S / I)=\operatorname{reg}_{A \otimes_{\mathbb{Z}} \mathbb{Q}}\left(A \otimes_{\mathbb{Z}} \mathbb{Q} / I_{A} \otimes_{\mathbb{Z}} \mathbb{Q}\right)=\operatorname{reg}_{A \otimes_{\mathbb{Z}} \mathbb{F}_{p}}\left(A / I_{A} \otimes_{\mathbb{Z}} \mathbb{F}_{p}\right)
$$

and $A / J \otimes_{\mathbb{Z}} \mathbb{F}_{p}$ is Gorenstein for $p \gg 0[\mathrm{HH}$, Theorem 2.3.5]. Then, the result follows from Theorem 5.6, because Stanley-Reisner rings in prime characteristic are $F$-pure.

\section{Acknowledgments}

We thank Carlos Espinosa-Valdéz and Delio Jaramillo-Velez for comments on an earlier draft. We also thank the referee for helpful comments.

\section{References}

[AE05] Ian M. Aberbach and Florian Enescu. The structure of F-pure rings. Math. Z., 250(4):791-806, 2005.

[DSNnB18] Alessandro De Stefani and Luis Núñez Betancourt. F-thresholds of graded rings. Nagoya Math. J., 229:141-168, 2018. 
[GSRTR02] M. González-Sarabia, C. Rentería, and H. Tapia-Recillas. ReedMuller-type codes over the Segre variety. Finite Fields Appl., 8(4):511-518, 2002.

[GW78] Shiro Goto and Keiichi Watanabe. On graded rings. I. J. Math. Soc. Japan, 30(2):179-213, 1978.

[HH] Melvin Hochster and Craig Huneke. Tight closure in equal characteristic zero. Unpublished manuscript available at http://www.math.lsa.umich.edu/ hochster/tcz.ps.

[HR76] Melvin Hochster and Joel L. Roberts. The purity of the Frobenius and local cohomology. Adv. Math., 21(2):117-172, 1976.

[HS06] Craig Huneke and Irena Swanson. Integral closure of ideals, rings, and modules, volume 336 of London Mathematical Society Lecture Note Series. Cambridge University Press, Cambridge, 2006.

[JV21] Delio Jaramillo and Rafael H. Villarreal. The v-number of edge ideals. J. Combin. Theory Ser. A, 177:105310, 35, 2021.

[MBPV17] José Martínez-Bernal, Yuriko Pitones, and Rafael H. Villarreal. Minimum distance functions of graded ideals and Reed-Mullertype codes. J. Pure Appl. Algebra, 221(2):251-275, 2017.

[NnBPV18] Luis Núñez Betancourt, Yuriko Pitones, and Rafael H. Villarreal. Footprint and minimum distance functions. Commun. Korean Math. Soc., 33(1):85-101, 2018.

[RMSV11] Carlos Rentería-Márquez, Aron Simis, and Rafael H. Villarreal. Algebraic methods for parameterized codes and invariants of vanishing ideals over finite fields. Finite Fields Appl., 17(1):81-104, 2011.

[Sch10] Karl Schwede. Centers of F-purity. Math. Z., 265(3):687-714, 2010.

[TW04] Shunsuke Takagi and Kei-ichi Watanabe. On F-pure thresholds. J. Algebra, 282(1):278-297, 2004.

[Vil15] R. Villarreal. Monomial Algebras. Monographs and Research Notes in Mathematics. Chapman and Hall/CRC, second edition, 2015. 
Luis NÚÑEZ-BETANCOURT,

Centro de Investigación en Matemáticas,

Guanajuato, Gto., México.

Supported by CONACyT Grant 284598, Ctedras Marcos Moshinsky, and SNI,

Mexico.

Email: luisnub@cimat.mx

Yuriko PITONES,

Centro de Investigación en Matemáticas,

Guanajuato, Gto., México.

Supported by CONACyT Grant 427234 and CONACyT Postdoctoral Fellow-

ship 177609.

Email: yuriko.pitones@cimat.mx

Rafael H. VILLARREAL,

Departamento de Matemáticas,

Centro de Investigación y de Estudios Avanzados del IPN,

Apartado Postal 14-740,

07000 Mexico City, D.F.

Supported by SNI, México.

Email: vila@math.cinvestav.mx 